\title{
A STRANGE BOUNDED SMOOTH DOMAIN OF HOLOMORPHY
}

\author{
BY KLAS DIEDERICH AND JOHN ERIK FORNAESS \\ Communicated by I. M. Singer, August 5, 1975
}

Introduction. Let $\Omega \subset \mathbf{C}^{n}$ be a bounded pseudoconvex domain. Does $\bar{\Omega}$ have a neighborhood basis consisting of pseudoconvex domains? It is well known that the answer to this question is, in general, "no". But it has been an open problem, at least since 1933 when the fundamental paper [1] of $\mathrm{H}$. Behnke and P. Thullen appeared, whether the answer might be in the affirmative under the additional hypothesis of smoothness of the boundary $b \Omega$. The main purpose of this note is to give an example of a bounded pseudoconvex domain $\Omega_{1} \subset \mathrm{C}^{2}$ with smooth boundary that nevertheless does not have a Stein neighborhood basis. Additional hypotheses that guarantee the existence of such a basis are given in [3]. The constructed domain $\Omega_{1}$ has some more strange properties. In particular, the conjecture of R. O. Wells [5, Conjecture 3.1], does not hold for $\Omega_{1}$ (Theorem 2) and the boundary $b \Omega_{1}$ cannot be described by a smooth function with positive semidefinite Leviform on the whole $\mathrm{C}^{2}$ at each point $p \in \mathrm{b} \Omega_{1}$.

Together with the beautiful example of Kohn and Nirenberg [4], this domain $\Omega_{1}$ shows that bounded smooth domains in $\mathbf{C}^{n}$ can have quite different analytic properties than strictly pseudoconvex domains.

The proofs of the theorems announced in this note will be contained in a later paper of the authors.

Definition of $\Omega_{1}$. We fix a smooth function $\lambda: \mathbf{R} \rightarrow \mathbf{R}^{+} \cup\{0\}$ with the properties $\lambda(x)=0$ for $x \leqslant 0, \lambda^{\prime \prime}(x)>0$ for $x>0$, and such that $\lambda$ is "sufficiently" convex. For $r>1$ we define a family of smooth functions $\rho:(\mathbf{C}-\{0\}) \times \mathbf{C} \rightarrow \mathbf{R}$ by putting

$$
\rho_{r}(z, w)=\left|w+e^{i \ln z \bar{z}}\right|^{2}-1+\lambda\left(|z|^{-2}-1\right)+\lambda\left(|z|^{2}-r^{2}\right)
$$

and, finally, we take

$$
\Omega_{1, r}=\left\{(z, w) \in(\mathbf{C}-\{0\}) \times \mathbf{C} \mid \rho_{r}(z, w)<0\right\} .
$$

The basic properties of the sets $\Omega_{1, r}$ are:

LEMMA. For each fixed $r>1, \Omega_{1, r}$ is a bounded pseudoconvex domain in $\mathrm{C}^{2}$ with smooth boundary. The boundary $\mathrm{b} \Omega_{1, r}$ is not strictly pseudoconvex exactly on the annulus

AMS (MOS) subject classifications (1970). Primary 32F15; Secondary 32E25, 46J15.

Key words and phrases. Pseudoconvex domains, Stein neighborhoods, Nebenhuelle, holomorphic approximations, strictly plurisubharmonic exhaustion functions. 


$$
M_{r}=\{(z, w)|1 \leqslant| z \mid \leqslant r \text { and } w=0\} .
$$

Nebenhuelle. For what follows we take $r$ large enough and keep it fixed. We put $\Omega_{1}=\Omega_{1, r}$. Furthermore, we pick two real numbers $a, b$ such that $1 \leqslant a<b \leqslant r$ and $\ln b^{2}-\ln a^{2}=2 k \pi$ for some integer $k$. Let now $f$ be a holomorphic function on a neighborhood $U$ of $\bar{\Omega}_{1}$. Then we have $\{(z, w)|| z \mid=$ $\left.a,\left|w+e^{i \ln a^{2}}\right|<1+\epsilon\right\} \subset U$ and $\left\{(z, w)|| z|=b| w+,e^{i \ln b^{2}} \mid<1+\epsilon\right\} \subset U$ for a certain $\epsilon>0$, and also $\{(z, 0)|a<| z \mid<b\} \subset U$. Therefore, the function

$$
\hat{f}(z, w)=\frac{1}{2 \pi i} \int_{|\eta|=b} \frac{f(\eta, w)}{\eta-z} d \eta-\frac{1}{2 \pi i} \int_{|\eta|=a} \frac{f(\eta, w)}{\eta-z} d \eta
$$

is a holomorphic continuation of $f$ to the set $\{(z, w)|a<| z \mid<b$ and $\left.\left|w+e^{i \ln a^{2}}\right|<1+\epsilon\right\}$. By doing this for all possible choices of $a$ and $b$, one gets

THEOREM 1. The Nebenhuelle of $\Omega_{1}$, which is defined to be the interior of the intersection of all pseudoconvex domains containing $\bar{\Omega}_{1}$, contains the domain

$$
\hat{\Omega}_{1}=\Omega_{1} \cup\left\{(z, w)\left|e^{\pi}<\right| z\left|<e^{2 \pi},\right| w \mid<2\right\} .
$$

In particular, $\hat{\Omega}_{1}-\bar{\Omega}_{1} \neq \varnothing$ and $\bar{\Omega}_{1}$ does not have a Stein neighborhood basis.

The fact that the set of weakly pseudoconvex points on $b \Omega_{1}$ is an annulus and not a full disc is important, because it can be shown:

Proposition. Suppose $\Omega$ is a bounded pseudoconvex domain in $\mathbf{C}^{2}$ with smooth boundary and such that the set of weakly pseudoconvex points of $\mathrm{b} \Omega$ is the disc $\left\{(z, w) \in \mathrm{C}^{2}|| z \mid \leqslant 1\right.$ and $\left.w=0\right\}$. Then $\bar{\Omega}$ has a neighborhood basis of Stein domains.

The algebras $A^{k}\left(\Omega_{1}\right)$. We define for every nonnegative integer $k$ and $k=\infty$ and for any domain $\Omega$ in $\mathbf{C}^{n}$ the algebra $A^{k}(\Omega)$ as consisting of all $k$-times continuously differentiable functions on $\bar{\Omega}$ which are holomorphic on $\Omega$. One can find for every fixed $r>1$ a number $s=s(r)$ such that the function $w^{1 / s}$ has a well defined branch on $\Omega_{1, r}$. (In fact, if one writes $w^{1 / s}=u+i v$, one even may assume that $u>0$ and $|v|<u$ on $\Omega_{1, r}$. ) It follows that the function $g(z, w)=\exp \left(-1 / w^{1 / s}\right)$ belongs to $A^{\infty}\left(\Omega_{1, r}\right)$, and cannot be holomorphically continued into a full neighborhood of any point on $M_{r}$. This gives

THEOREM 2. The algebra $H\left(\bar{\Omega}_{1, r}\right)$ of holomorphic functions on $\bar{\Omega}_{1, r}$ is not dense in $A^{0}\left(\Omega_{1, r}\right)$ with respect to the sup-norm on $\bar{\Omega}_{1, r} \cdot(C f . R$. O. Wells [5], Čirka [2] and Diederich and Fornaess [3] .) 
Another interesting property of the algebras $A^{k}\left(\Omega_{1}\right)$ is

THEOREM 3. There exists a function $f \in A^{0}\left(\Omega_{1, r}\right)$ with $f \equiv 1$ on $M_{r}$ and $|f(z, w)|<1$ for all $(z, w) \in \bar{\Omega}_{1, r}-M_{r}$. However, no such peak functions exist in $A^{k}\left(\Omega_{1, r}\right)$ for $1 \leqslant k \leqslant \infty$.

Plurisubharmonic exhaustion functions. It has been asked (see for instance [5]), whether for every bounded pseudoconvex domain $\Omega \subset \mathbf{C}^{n}$ with smooth boundary there exists a $C^{\infty}$-function $\sigma$ in a certain neighborhood $U$ of b $\Omega$ with the following properties: (a) $\Omega \cap U=\{p \in U \mid \sigma(p)<0\}$, (b) $d \sigma(p) \neq 0$ for all $p \in U$, (c) the Leviform of $\sigma$ at any point $p$ on $\mathrm{b} \Omega$ is positive semidefinite on the whole $\mathbf{C}^{n}$ (not only on the holomorphic tangent space to $\mathrm{b} \Omega$ at $p$ ). This is, in general, not true because we have

THEOREM 4. There exists no defining function $\sigma$ for $\Omega_{1}$ with the properties (a)-(c).

Finally, the following statement shows that Theorem 1 in [3] is sharp with respect to the regularity property at the boundary of the constructed exhaustion functions:

THEOREM 5. For every integer $l \geqslant 1$ there exists a number $r=r(l)$ such that on $\Omega_{1, r}$ there is no plurisubharmonic function $\sigma$ with negative values, $\lim _{p \rightarrow \mathrm{b} \Omega_{1, r}} \sigma(p)=0$ and such that $\sigma^{l}$ is differentiable on $\Omega_{1}$ and $d\left(\sigma^{l}\right)(p) \neq 0$ for all $p \in \mathrm{b} \Omega_{1}$.

\section{REFERENCES}

1. H. Behnke and P. Thullen, Zur Theorie der Singularitäten der Funktionen mehrerer komplexen Veränderlichen. Das Konvergenzproblem der Regularitätshüllen, Math. Ann. 108 (1933), 91-104.

2. E. M. Čirka, Approximation by holomorphic functions on smooth manifolds in $\mathrm{C}^{n}$, Mat. Sb. 78 (120) (1969), 101-123 = Math. USSR Sb. 7 (1969), 95-113. MR 39 \#480.

3. K. Diederich and J. E. Fornaess, Exhaustion functions and Stein neighborhoods for smooth pseudoconvex domains, Proc. Nat. Acad. Sci. U.S.A. (to appear).

4. J. J. Kohn and L. Nirenberg, A pseudo-convex domain not admitting a holomorphic support function, Math. Ann. 201 (1973), 265-268. MR 48 \#8850.

5. R. O. Wells, Jr., Function theory on differentiable submanifolds, Contributions to Analysis, ed. L. V. Ahlfors et al, Academic Press, New York and London 1974, pp. 407-441.

DEPARTMENT OF MATHEMATICS, PRINCETON UNIVERSITY, PRINCETON, NEW JERSEY 08540 